\title{
Model-Centered Instruction, the Design, and the Designer
}

Andrew S. Gibbons III

Brigham Young University, andy_gibbons@byu.edu

Follow this and additional works at: https://scholarsarchive.byu.edu/facpub

Part of the Educational Psychology Commons, and the Educational Technology Commons

\section{Original Publication Citation}

Gibbons, A. S. (2008). Model-Centered Instruction, the Design, and the Designer. In D. Ifenthaler, P. Pirnay-Dummer, and J. M. Spector (Eds.), Understanding Models for Learning and Instruction. New York: Springer Science+Business, 161-174.

\section{BYU ScholarsArchive Citation}

Gibbons, Andrew S. III, "Model-Centered Instruction, the Design, and the Designer" (2008). Faculty Publications. 4656.

https://scholarsarchive.byu.edu/facpub/4656

This Book Chapter is brought to you for free and open access by BYU ScholarsArchive. It has been accepted for inclusion in Faculty Publications by an authorized administrator of BYU ScholarsArchive. For more information, please contact ellen_amatangelo@byu.edu. 


\title{
Model-Centered Instruction, the Design, and the Designer
}

\author{
Andrew S. Gibbons, Brigham Young University
}

\begin{abstract}
A model of instruction described by Wenger (1987) identifies three elements that are active during instruction: the mental model the instructor wishes to share with the learner, the external experience used to communicate the mental model, and the evolving mental model of the learner. Gibbons (2003a), writing in response to Seel (2003), noted this three-part description as a bridge concept relating learning and instruction.

This view has important practical implications for designers of instruction. For example, Gibbons and Rogers (in press) propose that there exists a natural layered architecture within instructional designs that corresponds with instructional functions. Among these layers is the content layer, which determines the structural form in which learnable subject-matter is stored and supplied to the learner. This may include the expression of the content in terms of tasks, semantic networks, rules, or other structures. The designer's commitment at the content layer strongly constrains all other parts of the design, making some future decisions imperative, some irrelevant, and defining the range of possibilities for still others.

One possible content layer commitment is to select the model structure as the basic unit of analysis. Having made the model the primary content structure commitment influences designer choices within other layers. This chapter describes the implications
\end{abstract}


for designers of a model content commitment. It describes the constraints automatically placed on other layers of the design.

Keywords: Model-centered instruction; instructional design; design layers; design languages. 


\title{
Model-Centered Instruction, the Design, and the Designer
}

\author{
Andrew S. Gibbons \\ Brigham Young University
}

This chapter discusses implications for the structures included in an instructional design when the subject-matter consists of a model of a dynamic system. Wenger (1987) identifies three elements that are active in such instruction: the mental model the instructor wishes to share with the learner, the external experience used to communicate the mental model, and the evolving mental model of the learner: "Of central importance...is the notion of model: the model of the domain, model of the student, and model of the communication processes..." (p. 7). According to Wenger, this model of instruction leads to a "radical” methodological shift for designers: “...The primary goal becomes computational understanding of processes of knowledge communication rather than an optimization of the production of systems." As a result, he says, the designer's problem becomes creating a representation of knowledge, which eventually results in "a mapping of knowledge into a physical medium" (p. 312).

This mapping of conceptual content which exists only in the mind of the designer or the subject-matter expert onto a physical medium creates a subtle distinction, which Wenger feels has much practical significance.

It is useful to be able to speak about the knowledge that is the source of this mapping, and we will use the adjective epistemic to refer to this 'disembodied' level. Whether such an epistemic level really exists in some 
Platonic sense is not the point here. The claim is, rather, that the distinction between the epistemic and the representational levels is useful in designing and evaluating models of communicable knowledge. (p. 312, emphasis in the original)

Wenger's isolation of the representational (mediated) model has further implications when we consider that there are two ways in which the computer medium can represent the knowledge: on the one hand invisibly within an information-processing engine, and on the other hand as sensations at a sensory surface where the learner can experience the model.

In fact it is a distinction between two forms of representation of the knowledge to be conveyed; we will simply call these the internal and external representations, respectively. ... This perspective is a useful one for tutoring systems. Indeed, not only does the interface language map meaning onto a system of symbols, but this 'external representation' can actually compete with the internal representation as a vessel for domain knowledge (p. 312, emphasis in the original).

\section{Model-Centered Instruction}

Gibbons (2003a), commenting on Seel (2003), supported Wenger's distinction between the expert's and the learner's mental models and the "experience used to communicate the model". This distinction presents a challenging design problem for which designers have few formal design concepts. According to Gibbons, "the question of interest...is design structure. How do we harness and focus the structuring principle of the state-changing model — not only as an influence on the type of knowledge learned, but 
as an influence on the nature and structure of the design itself" (p. 296). This influence is felt in many parts of the design:

Designers need (but do not currently have) the ability to describe classes of design architecture and discuss them in professional discourse. Design architecture here does not refer to software architecture or instructional strategy architecture: it refers to the architecture of the entire design, but most importantly to the central structural element of the design: those structures of the design that are decided first, that determine and shape the rest of the design, and that do not change as details and finish are added to a design (p. 296).

Gibbons developed a design theory of model-centered instruction (2001) for the purpose of exploring the design implications of dynamic-model content. Model-centered instruction is instruction that is carried out through interaction with dynamic models, and the experience with the model is supplemented by the activities of a learning companion that may supply a variety of coaching, feedback, and other learning support services. Varieties of model-centered instruction are created by considering all of the variations of this basic configuration.

In a model-centered design the initial commitment to the dynamic model as the "central structural element" of a design places constraints on those design decisions that follow it. These constraints remain in force as long as that original commitment to dynamic modeling is maintained. Among these constraints are:

- Constraints on the type and execution of instructional strategy employed as an augmentation of the learner's experience interacting with the model. 
- Constraints on the types and actions of controls given to the learner for managing the model experience.

- Constraints the kinds of message that can pass from the model and its augmentations to the learner.

- Constraints on the representation of the model to the learner (what Wenger would call the "external" representation).

- Constraints on the media logic used to execute the model and its augmentations.

- Constraints on the collection and use of data generated during the model experience.

"Constraint" is used here in the sense that Stokes (2005) described, in which a constraint is both a limitation (a closing off of certain options) and an opportunity for innovation (an opening of new options).

\section{Model-Centered Instruction and Simulation}

To this point, the reader may have assumed that the terms "simulation" and "model-centered instruction" are synonymous. However, I do not believe that these terms should be used interchangeably. Each represents a way of viewing the assumptions of an instructional design from a particular perspective. Simulation (of the instructional variety) usually refers to an external representation and a type of experience afforded to a learner. A learner is said to "use" a simulation. Model-centered instruction refers to a product and experience architecture that involves many layers of organization, some visible and some completely invisible. Implicit in a model-centered architecture is a commitment to one or more abstract dynamic models (Wenger's internal model) which must be represented to a 
learner (by Wenger's external model) in a way that communicates the essential aspects of the expert's model to a learner, who uses the communication in the construction of personal knowledge.

An example of this is an instructional methodology described by Brown and Palincsar (1989) as reciprocal teaching. The core activity of reciprocal teaching is the use of pre-assigned questions asked by learners as a means of mining a text reading or a shared observational experience in order to comprehend its meaning. The details of how this is accomplished are not as important to the present purpose as is the statement of the principle by which reciprocal teaching works. In describing this, Brown and Palincsar name the activities and then identify the operational principle of reciprocal teaching: ...These...strategic activities...structure intrapersonal as well as social dialogues. Reviewing content (summarizing), attempting to resolve misunderstandings (clarifying), anticipating possible future text development (predicting), and assessing the state of one's gradually accumulating knowledge (questioning) are all activities that the experienced learner engages in while studying independently, by means of an internal dialogue. The reciprocal teaching procedure renders such internal attempts at understanding external. Reciprocal teaching provides social support during the inchoate stages of the development of internal dialogues. In the course of repeated practice such meaning-extending activities, first practiced socially, are gradually adopted as part of the learner's personal repertoire of learning strategies (p. 415, emphasis in the original). 
Reciprocal teaching relies on the choreographed joint activities of several learners to produce a visible model of comprehension which, if observed and experienced repeatedly by a learner may be internalized and used as a personal comprehension process. Since this dynamic model of that comprehension process constitutes the key subject-matter to be learned, reciprocal teaching is a design configuration that can be termed "model-centered". All design decisions that follow after the model decision revolve around this central commitment and are conditioned by it. Should some other design factor be given higher priority in the design, it would become strategy-centered, message-centered, or media-centered instead (see Gibbons, 2003b). Gibbons and Fairweather (2000) describe several innovative designs emerging from research which are quite diverse in their surface features but that share an underlying model-centered design architecture. Contrasting simulation and model-centered instruction clarifies the important point that model-centered instruction focuses attention on the entire architecture of the design, placing priority on the model as a structural foundation. The remainder of this chapter describes the impact of the commitment to model-centering on the remaining layers of a design. In this discussion, the reader should keep in mind that though true simulations have a model-centered architecture, many members of the class of model-centered designs (reciprocal teaching being an example) do not look on the surface like simulations and would be construed by many as not being simulations.

\section{Design Layers}

Gibbons and Rogers (in press) describe instructional designs structurally, providing a way to consider the "remaining layers of design". A layered instructional design sees the total design problem in terms of many individual sub-problems: 
- Every instructional design must solve a representation problem by providing specifications for the part of the instructional artifact that can be sensed (through sight, sound, touch, smell, etc.) by exposure to media or realia. (This is Wenger's external model.)

- Every instructional design must solve a strategy problem by describing the patterns of tutorial conversational exchanges that can be engaged in between learner and instruction source, the setting in which they take place, and the social configuration and roles of all participants.

- Every instructional design must solve a messaging problem by providing a description of the system of individual messages that can be communicated from the instruction source, in service of the instructional strategy, and for the purpose of driving the selection or construction of representations to the learner. The solution to the messaging problem supplies a bridge between abstractions in the strategy layer and concretions in the representation layer.

- Every instructional design must solve a control problem by specifying the communication controls and related symbols through which the learner will be able to communicate choices, responses, and strategic control over the instructional source.

- Every instructional design must solve a media-logic problem by describing the manner in which the functions of all of the other layers will be enacted by humans, instructional media, or some combination of both.

- Every instructional design must solve a data management problem by describing the elements of data from the instructional interaction that will be 
captured, recorded, stored, analyzed, reported, and used to influence the ongoing course of the instructional interaction.

\section{Content Layer Constraints in Model-Centered Designs}

The only layer of the design problem that is addressed by a commitment to model-centering is the content problem: in model-centered instruction the content (which includes model state data, subject-matter information, and dynamic change information) is supplied through computation of changing model states. Model-centered instruction assumes that learners will be enabled to observe and interact with three types of dynamic model: (1) models of cause-effect systems, (2) models of performance with respect to those systems, and (3) models of environments that influence either the performance or the cause-effect systems. It assumes that learners will either observe the operation of models or perform operations on models to observe the effects. Selection of the appropriate model or combination of models is critically important. Bransford et al, (2000), asserts that "one is struck by the complexity of selecting appropriate models for particular mathematical ideas and processes" (p. 168). A designer must avoid unnecessarily complex models which have variables that are of no consequence to the learner and must be careful to select a model that leads to the desired processing by the learner.

The commitment to the model at the content layer of the design imposes limitations and provides opportunities (both of which can be considered constraints) at all other layers of the design. The sections that follow describe some of these. 


\section{Strategy Layer Constraints in Model-Centered Designs}

Models themselves can only supply information on changing model states. The model itself produces no commentary on its own actions, no insight into the play of forces in its inner workings, and no scaffolds to support incremental learning (see, for instance, Clancey, 1984a). It is possible to learn from an unaugmented model by observing and experimenting with it, but the efficiency of such learning is low and can lead to misconceptions. Therefore, most instructional model experiences are accompanied by supports that assist the learner during observation and operation of the model (Gibbons, 2001; Gibbons et al., 1997). The strategic design principles described in this section do not comprise a complete list of scaffolding augmentations for modelcentered instruction. The ones included have been chosen to illustrate the important structural differences implied by a decision to use a model-centered design architecture. Additional types of augmentation during model experience are described by Gibbons et al (1997).

One type of augmentation includes supplying one or more problems to frame the learner's exposure to and interaction with the model (Gibbons, 2001). The problem serves as a lens and a mask during the model experience. As a lens, problems stimulate learner interaction with and observation of model details. As a mask, problems focus interaction on only selected relationships within the complete model, allowing relationships of immediate interest to be foregrounded for consideration. The learner can either solve a problem or observe a problem being solved as a means of exposing key relationship information of momentary interest. Problems may take a number of forms, but a model-centered commitment implies that problems of some type will be used. 
Model experience in the absence of problems is a possibility, but in such unaugmented explorations it can be seen that the learner becomes the problem poser and that actions toward the model in service of learning are evidence of self-posed problems (or questions), even if they consist of something as simple as "What happens when I press this button?". The design of the model can be influenced by the types of problem the designer intends to use.

A second type of strategic model augmentation consists of initiative sharing in the selection roles and goals. Gibbons (2001) describes several strategic decisions learners may share or fully control:

- Role negotiation (observer, participant, main agent, exclusive agent)

- Initiative negotiation (learner, instruction source, shared)

- Performance goal selection (at any of several levels of granularity)

- Problem selection (at any of several levels of granularity)

- Strategic goal selection (for problem solving approach)

- Means selection/design (for strategic goals selected)

- Means execution

- Evaluation of goal achievement

A third type of augmentation used to supplement model experience consists of conversational tutorial messaging support during model interaction. Messaging is discussed in more detail in a later section. Strategically, however, it is important to note that familiar structures of exposition and information-delivery that are used in the design of more traditional forms of instruction are subordinated in model-centered instruction. Model-centering does not encourage the use of long information presentations, so the 
designer must think more in terms of the conversation the learner is having with the model, expressed through choices and interactions.

\section{Control Layer Constraints in Model-Centered Designs}

The design of control systems takes on special importance in model-centered designs. Controls of several types are required in the less-structured environment created by model-centered instruction. Gibbons et al (in press) names them:

...Sets of special-purpose controls that serve needs related to several simulation functions: (1) controls that allow the learner to act upon the model, (2) controls that adjust patterns of augmentation, (3) controls that adjust the representation of the model or the viewpoint from which the learner can observe the presentation, and (4) controls over personal data reporting for monitoring outcomes, performance, progress, trends, history, and scheduling.

Learners use controls to convey messages to the instructional source. In combination, the control and messaging systems provide the two-way communication channel through which learner and instructional source communicate. Controls and messaging are thus the medium through which interactions proceed. In traditional instructional forms, control systems are so standard that they tend to fuse with other aspects of the design. In a model-centered design, control systems must be invented which are related to the characteristics of the content model(s), the support functions, and conversational patterns of the strategic augmentations, so they tend to be more customized. Crawford (2003) suggests that the beginning point of the design of such 
control systems is to define the "verbs" that represent actions the learner can take during interactions.

\section{Message Layer Constraints in Model-Centered Designs}

The instructional conversation referred to earlier takes its structure from the strategic instructional augmentations of the strategy, but the expression of a strategy as a conversation sometimes entails a complex pattern of learner-to-instruction exchanges. Message layer structures provide an intermediate mapping entity that allows the larger intentions of the strategy to be expressed in terms of smaller particles of expression. There are many examples of message structuring systems in the literature, including Merrill's Component Display Theory (1994), Horn's information mapping (1997), systems for the recording and analysis of classroom conversation (Simon \& Boyer, 1974), and more recent ones for analysis of peer-to-instructor and peer-to-peer conversation (Sawyer, 2006).

Message structures provide the possibility of flexible, unfolding patterns of communication for a single strategic intention. Moreover, they allow a single communication intention to map onto multiple representation forms. Other kinds of communication must also be provided for in addition to the strategic ones. Messages must be conveyed to the learner about control availability, access to personal learning data (progress, scores, etc.), and access to alternative representation modes, such as different perspective or representation style (schematized, literalized, abstracted, etc.).

The design for a messaging system centers around an ontology of message types and a catalogue of messaging patterns. The first concern of message layer design for model-centered instruction is to enumerate the basic patterns of moment-to-moment 
exchange that the designer feels will permit the expression of the full range of strategic, control, data, and representation information. The designer also must define the rules for interpreting messages, such as those described by Fox (1993) for technology-based media:

- Interruptions by the learner should be possible

- Thing reference (pointing) and object sharing should be more formalized

- Silences should be flagged with intent

- Communication of backchannel cues (emotional states, body language, attitude) should be facilitated

- Multiple sequential messages should be possible from the same speaker without a break (e.g., musings "aloud”)

- Short delays in correction might be deliberately used to signal to the student the need to think and respond again

- Ways should be found to make the learner's process actions (thinking) known to the tutor. When message design has been executed, the designer has the core of a mechanism by which instructional messages can be generated dynamically using computed data from the model, the strategic function, and other sources. This was an early goal of some intelligent tutoring systems (Carbonell, 1970; Collins et al, 1975; Clancey, 1984b). More recently, Drake et al (1998) have used a messaging design to allow a simulation to generate messages and representations from a combination of computed data and primitive message fragments during presentation, demonstration, and practice stages of instruction. 


\section{Representation Layer Constraints in Model-Centered Designs}

Up to the present, the representations- - the sensed surface elements of instruction - for both live and technology-based instruction have tended to be static and unchanging, with a relatively small seasoning of dynamic ones. Since model-centered instruction is grounded in the principle of making it possible for the learner to sense state changes, forces, and trends of change, this typical balance between static and dynamic representations is reversed. Moreover, what is represented changes as well. The most common (and affordable) tradition has been to show static 2-dimensional opaque surfaces superimposed with static symbolic enhancements (arrows, auras, etc.) intended to illustrate flow and dynamism. Model-centered instruction favors dynamic 4-dimensional effects incorporating integral dynamic symbolic elements that illustrate changes in multiple invisible forces at once. This constraint is important because it is the dynamic operation of invisible forces that most often constitutes the basis for understanding dynamic models.

Model-centering introduces new terms into the representation lexicon for designers used to traditional and low-cost approaches to representation. Designers must consider refresh rates, strict synchronization of multimedia events, multi-perspective views, intelligent display assembly and coordination, storage and controlled replay of representation event sequences, correlation and synchronization of stylistic modes (schematic, literal, metaphorical, etc.), time and space warping (slow-down, speed-up magnification, diminution, zooming), navigation, time-trace representation, and multiple message-to-representation mappings. Rather than thinking of representation resources as stored, pre-composed, static elements, the model-centered designer thinks in terms of 
data-driven, generated media experiences where possible and families of well-crafted animation sequences where it is not.

\section{Media-Logic Layer Constraints in Model-Centered Designs}

Media-logic consists of the set of rules necessary to stage the events incorporated in a design. Media-logic is an essential element of live instruction as well as technologybased instruction; it generates the sequence of events during instruction. For modelcentered instruction of all kinds it consists of algorithms, heuristics, or explicit instructions used by a computer or a human instructor that direct how to make instructional events occur. Media-logic should not be confused with the strategic decision-making function (within the strategy layer) that determines which events might take place. Gibbons et al (2001) describes media-logic as "the place where the designer's abstract instructional constructs and concrete logic constructs [of] the development tool come together".

Media-logic executes models, executes augmentation computations, executes message formation, executes representations, accepts control input, and executes datarelated functions. Moreover, it integrates and coordinates the order of computation for these functions. (Keep in mind that "computation" here includes human instructor decision making and judgment.) This integration and coordination most frequently takes the form of a continuous cycle of activities, many of which can occur in a parallel sequence, where that is possible. Baldwin and Clark (2000) describe the economics of functional modularization with respect to logic functions. Munro et al (2006) demonstrates that this principle applies to model-centered forms of instruction. 


\section{Data Management Layer Constraint in Model-Centered Designs}

Model-centered designs can make much different use of data management functions than non-model-centered instructional forms. Because model-centered instruction entails learner interactions within a dynamic context, it is possible for modelcentered instruction to generate much larger volumes of data from an instructional encounter. Moreover, that data can be interpreted with respect to the momentary state changes within that context. An action at Time A can be interpreted as having a particular meaning in terms of the learner's knowledge; the same action at Time B may have a much different interpretation.

Because the data generated during such interactions is interpretable and can be used in future instructional decisions, much more data can be captured. In some cases, the volume of this data prohibits immediate processing, so provision for data storage, eventual analysis, and visualization becomes an important design factor. Following the analysis of this volume of data, it may not be possible to describe the performance within the environment in terms of a few simple indices. Because of this, model-centered instructional designs can challenge the most widely-held metaphors of instructional management systems as simply score repositories. Where the volume of data does allow immediate processing, the results of processing can be reported to strategic functions that use the results to make real-time modifications to the model and its augmenting processes.

These considerations make the design of data management more involved. The designer must consider when and how often data will be collected, the granularity of data recorded, the interpretation rules (whether the data is processed immediately or after a 
delay), and the use of the results of interpretation, both by the learner, and by the instructional source.

\section{Conclusion}

The purpose of this chapter is to demonstrate the effects that ripple through the many layers of a design when a specific commitment is made within one layer of the design. Using proposals by Wenger (2003) and Gibbons (2003a) that content can be described in terms of dynamic models, I have traced the implications of a commitment to model content within the strategy, control, message, representation, media-logic, and data management layers of the design. This analysis has highlighted the many differences within each layer of the design attendant to the content decision. A similar analysis based on a different content commitment or a similar commitment within any of the other layers would demonstrate the same result: any layer of a design is sensitive to decisions made in other layers, and decisions made within one layer constrain decisions within other layers, either by eliminating or creating design possibilities.

This finding should stimulate designers to examine more carefully the assumptions that are often built into their designs. It should also lead to more detailed examination of classes of design, of which model-centered is but one example. I propose that doing so may result in the recognition of distinct classes of design that are based on underlying structural differences rather than on surface appearances. Such a perspective encourages thinking about designs and the creation of designs in a new light—one that sees the abstract operational principle of a design as being a tool for generating not just individual designs but whole new families of designs that may appear much different on the surface but owe their genesis to a similar underlying architecture. 


\section{References}

Baldwin, C. Y., \& Clark, K. B. (2000). Design rules, vol. 1: The power of modularity. Cambridge, MA: MIT Press.

Bransford, J. D., Brown, A. L., Cocking, R. R. (Eds.). (2000). How people learn: Brain, mind, experience, and school. Washington, D. C.: National Academy Press.

Brown, A. L., \& Palincsar, A. S. (1989). Guided, cooperative learning and individual knowledge acquisition. In L. Resnick (Ed.), Knowing, learning, and instruction: Essays in honor of Robert Glaser. Hillsdale, NJ: Lawrence Erlbaum Associates.

Carbonell, J. (1970). AI in CAI: An artificial intelligence approach to computer-assisted instruction. IEEE transactions on man-machine systems, 11, 190-202.

Clancey, W. J. (1984a). Extensions to rules for explanation and tutoring. In B. G. Buchanan \& E. H. Shortliffe (Eds.), Rule-based expert systems: The MYCIN experiments of the Stanford heuristic programming project. Reading, MA: Addison-Wesley.

Clancey, W. J. (1984b). Use of MYCIN's rules for tutoring. In B. G. Buchanan \& E. H. Shortliffe (Eds.), Rule-based expert systems: The MYCIN experiments of the Stanford heuristic programming project. Reading, MA: Addison-Wesley.

Collins, A., Warnock, E., \& Passafiume, J (1975). Analysis and synthesis of tutorial dialogues. In G. Bower (Ed.), The psychology of learning and motivation (Vol. $I X)$. New York: Academic Press.

Crawford, C. (2003). The art of interactive design. San Francisco, CA: No Starch Press.

Drake, L., Mills, R., Lawless, K., Curry, J., \& Merrill, M. D. (1998). The role of explanations in learning environments. Paper presented at the annual meeting of the American Educational Research Association, San Diego, CA.

Fox, B. A. (1993). The human tutorial dialogue project: Issues in the design of instructional systems. Hillsdale, NJ: Lawrence Erlbaum Associates.

Gibbons, A. (2001). Model-centered instruction. Journal of structural learning and intelligent systems, 14(4), 511-540.

Gibbons, A. S. (2003a). Model-centered learning and instruction: Comments on Seel (2003). Technology, instruction, cognition, and learning, 1(3), 291-9.

Gibbons, A. S. (2003b). What and how do designers design?: A theory of design structure. Tech trends, 47(5), 22-27.

Gibbons, A. S., \& Fairweather, P. G. (2000). Computer-based instruction. In S. Tobias \& J. D. Fletcher (Eds.), Training and retraining: A handbook for business, industry, government, and the military. New York: Macmillan Reference USA.

Gibbons, A. S., Fairweather, P. G., Anderson, T., \& Merrill, M. D. (1997). Simulation and computer-based instruction: A future view. In C. R. Dills \& A. J. Romiszowski (Eds.), Instructional development paradigms (pp. 769-805). Englewood Cliffs, NJ: Educational Technology Publications.

Gibbons, A. S., Lawless, K. A., Anderson, T. A., \& Duffin, J. R. (2001). The web and model-centered instruction. In B. R. Khan (Ed.), Web-based training. Englewood Cliffs, NJ: Educational Technology Publications.

Gibbons, A. S., McConkie, M., Seo, K. K., \& Wiley, D. (in press). Theory for the design of instructional simulations and microworlds. In C. M. Reigeluth \& A. Carr- 
Chellman (Eds.), Instructional-design theories and models, Volume III. Mahwah, NJ: Erlbaum.

Gibbons, A. S. \& Rogers, P. C. (in press). The architecture of instructional theory. In C. M. Reigeluth \& A. Carr-Chellman (Eds.), Instructional-design theories and models, Volume III. Mahwah, NJ: Erlbaum.

Horn, R. E. (1997). Structured writing as a paradigm. In C. R. Dills \& A. J. Romiszowski (Eds.), Instructional development paradigms. Englewood Cliffs, NJ: Educational Technology Publications.

Merrill, M. D. (1994). The descriptive component display theory. In M. D. Merrill \& D. G. Twitchell (Eds.), Instructional design theory. Englewood Cliffs, NJ: Educational Technology Publications.

Munro, A., Surmon, D. \& Pizzini, Q. (2006). Teaching procedural knowledge in distance learning environments. In R. Perez \& H. O'Neal (Eds.), Web-based learning: theory, research, and practice. Mahwah, NJ: Lawrence Erlbaum Associates.

Sawyer, R. K. (2006). Analyzing collaborative discourse. In R. K. Sawyer (Ed.), The Cambridge handbook of the learning sciences. Cambridge, UK: Cambridge University Press.

Seel (2003). Model-centered learning and instruction. Technology, instruction, cognition, and learning, 1(1), 59-85.

Simon, A., \& Boyer, E. G. (1974). Mirrors for behavior III: An anthology of observation instruments. Wyncote, PA: Communication Materials Center in Cooperation with Humanizing Learning Program, Research For Better Schools, Inc.

Stokes, P. (2005). Creativity from constraints: The psychology of breakthrough. New York: Springer.

Wenger, E. (1987). Artificial intelligence and tutoring systems. Los Altos, CA: Morgan Kauffmann Publishers. 